\section{Was Patienten fragen}

Onkologe 2021 $27: 77-78$

https://doi.org/10.1007/s00761-020-00892-6

Online publiziert: 21. Dezember 2020

(c) Springer Medizin Verlag GmbH, ein Teil von

Springer Nature 2020

Andrea Gaisser

Heidelberg, Deutschland

\title{
Die Coronapandemie und wie Krebspatienten sie erleben
}

die Versorgung der schwer an COVID-19 Erkrankten Auswirkungen: Nicht dringend erforderliche Untersuchungen, teilweise auch Behandlungen wurden ggf. verschoben, Nachsorgetermine ausgesetzt, um Krebspatienten nicht unnötig durch das Aufsuchen von Praxis oder Klinik zu gefährden. Umgekehrt vermieden viele Patienten aus Furcht vor Ansteckung von sich aus Arztbesuche. Auch dazu erhielt der Krebsinformationsdienst zahlreiche Anfragen: Meine Chemotherapie wurde verschoben, ist das schlimm? Kann ich die Anschluss-Reha auf später verlegen? Soll ich wegen des Ansteckungsrisikos Arztoder Kliniktermine absagen? Soll ich meine Nachsorge wahrnehmen?

Die Tendenz zur Vermeidung von Arztbesuchen betraf auch Untersuchungen zur Krebsfrüherkennung und zur Abklärung von Symptomen. Eine vom Deutschen Krebsforschungszentrum, der Deutschen Krebshilfe und der Deutschen Krebsgesellschaft gebildete Task Force zur Beobachtung der Versorgungslage warnte im Frühjahr nach entsprechenden Berichten aus der Ärzteschaft besonders davor, solche Untersuchungen aufzuschieben - dies könne eine Bugwelle von zu spät diagnostizierten Krebsfällen aufbauen. Die Task Force hat ihre Arbeit und das Monitoring der Versorgungssituation in der Onkologie während der zweiten Welle wiederaufgenommen. Eine Zusammenfassung der Erfahrungen aus der ersten Pandemiewelle wurde vor Kurzem publiziert [1].

Der Lockdown schließlich hatte beträchtliche Auswirkungen auf die soziale Situation von Patienten: Wie ist die Gefährdung im familiären Zusammenleben? Sollten im Haushalt lebende Personen mit intensiven Außenkontakten besser ausziehen? Kann ich einkaufen gehen? Kann ich Besuch bekommen, Freunde besuchen? Was mache ich mit den Kindern? Können, sollen sie in die Schule, die Kita? Worauf müssen Angehörige achten?

Ab April 2020 hat der Krebsinformationsdienst die zentralen Themen der Anfragen zu SARS-CoV-2 und COVID-19 dokumentiert. Die Rangfolge zeigt - Abb. 1.

Viele der Unklarheiten und Unsicherheiten aus den ersten Monaten der Pandemie sind mittlerweile ausgeräumt. Das ten und realen Risiken besonders in den ersten Monaten der Pandemie hautnah erfahren - der Dienst wurde phasenweise von Anfragen förmlich überrannt:

Bin ich mit meiner Erkrankung (besonders) gefährdet für einen schweren Verlauf von COVID-19? Ist mein Immunsystem durch die Krebstherapie geschwächt? Wie kann ich mich schützen? Worauf müssen Angehörige achten? Wie soll ich mich verhalten? Kann ich, soll ich mich testen lassen? Muss ich als Risikopatient zur Arbeit gehen? Habe ich ein Recht auf Homeoffice? Habe ich Anspruch auf ein Taxi statt ÖPNV, um zum Arzt zu kommen? ...

Auch auf die onkologische Versorgung hatten die Fokussierung auf Corona und die „Reservierung“" von Ressourcen für

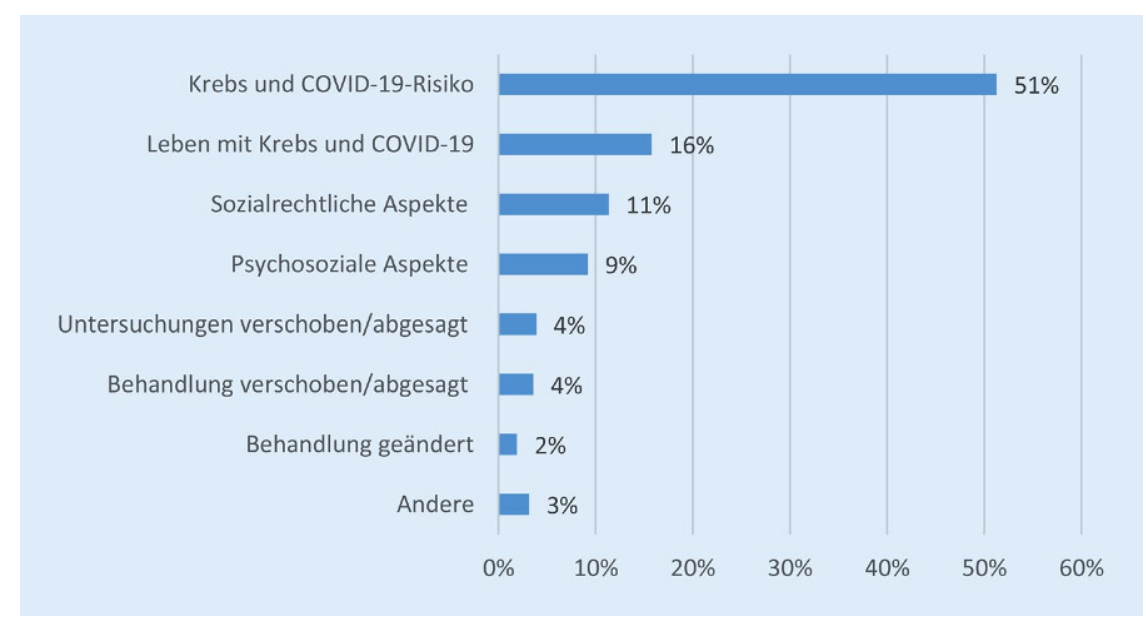

Abb. 1 ॥ Themenranking der "Coronafragen“ an den Krebsinformationsdienst ( $n=1598$ Einzelfragen) 
Wissen um den Erreger, um Risikofaktoren, Präventionsmaßnahmen und um die COVID-19-Erkrankung selbst ist in der Zwischenzeit rasant gewachsen, Versorgungsstrukturen wurden angepasst, Behandlungsmöglichkeiten verbessert. Entsprechend sind die Anfragen zum Thema mittlerweile zurückgegangen, aber der Krebsinformationsdienst gibt bei Bedarf weiterhin telefonisch und per E-Mail Auskunft zu allen individuellen Fragen im $\mathrm{Zu}$ sammenhang mit dem Coronavirus und Krebs und hat Antworten auf die häufigsten Fragen auch auf seinen Internetseiten zusammengestellt (https://www.krebsinformationsdienst.de/leben/alltag/coronavirus-krebs-haeufige-fragen.php).

Denn die Pandemie hat weiter Auswirkungen - psychisch, sozial und für viele auch finanziell durch Kurzarbeit oder Jobbzw. Auftragsverlust. Und die Infektionszahlen sind seit Oktober wieder rasant angestiegen, die zweite Welle und der zweite Lockdown sind da, mit neuerlichen Einschränkungen und Unsicherheiten. Neben der adäquaten Versorgung schwer an COVID-19 Erkrankter muss auch jetzt wieder darauf geachtet werden, dass für alle, und ganz besonders für gefährdete Gruppen und Patienten mit schweren Erkrankungen, eine gute, sichere Gesundheitsversorgung gewährleistet wird.

\section{Befragung von Patienten}

Im Rahmen der fightCOVID@DKFZInitiative forschen in Heidelberg seit dem Frühjahr zahlreiche wissenschaftliche Gruppen zu wichtigen Fragestellungen im Zusammenhang mit SARS-CoV-2 und COVID-19. Neben medizinisch-naturwissenschaftlichen Themen gilt das Interesse auch der Patientenversorgung und Belastungen der Betroffenen durch die Situation. Ein Projekt untersucht mit einer Befragungsstudie die Auswirkungen der Pandemie für Krebspatienten. Ein eigens dafür entwickelter Fragebogen unter Einbeziehung validierter Instrumente soll die Erfahrungen Betroffener genauer beleuchten:

- Hat sich durch die Pandemie etwas in der Versorgung und Betreuung geändert? Und wenn ja, was?

- Wie sind das allgemeine Befinden und die seelische Verfassung der Pa- tienten? Fühlen sie sich durch die Pandemiesituation zusätzlich belastet?

- Haben sie durch die Coronapandemie besondere finanzielle Belastungen oder Einbußen?

Seit Mitte Juli läuft als Pilot eine Querschnittsstudie beim Krebsinformationsdienst, die gemeinsam mit der Abteilung Gesundheitsökonomie, der Arbeitsgruppe Cancer Survivorship und dem Epidemiologischen Krebsregister Baden-Württemberg am Deutschen Krebsforschungszentrum sowie dem Schwerpunkt Ethik und patientenorientierte Versorgung des Nationalen Centrums für Tumorerkrankungen konzipiert wurde. $\mathrm{Zu}$ der anonymen Onlinebefragung werden Patienten eingeladen, die sich an den E-Mail-Service des Diensts wenden. Aus den bisherigen Rückläufen geht hervor, dass zwar nur wenige Patienten von Änderungen bei der antitumoralen Therapie betroffen waren. Verschiebungen von Kontroll- und Nachsorgeterminen wurden allerdings häufiger angegeben. Und ein beträchtlicher Teil der Befragten macht sich Sorgen über die Qualität ihrer medizinischen Versorgung unter Pandemiebedingungen. Auch ist die Angst, selbst an COVID-19 zu erkranken, und mehr noch die Angst um Angehörige und Freunde ein großes Thema. Noch mehr machen vielen Betroffenen die Beschränkungen persönlicher Kontakte zu schaffen. Nur etwa jeder Fünfte gab an, sich durch die Situation gar nicht beeinträchtigt zu fühlen. Nachdem die Befragung in der abklingenden ersten Welle begonnen wurde, der ein relativ "normaler“ Sommer folgte, bildet sie nun die zweite Welle der Pandemie ab.

Folgen sollen in Zusammenarbeit mit dem Krebsregister Baden-Württemberg weitere bevölkerungsrepräsentative Erhebungen bei im Register erfassten Patientinnen und Patienten mit Brustkrebs, Darmkrebs, Lungenkrebs, Prostatakrebs und hämatoonkologischen Erkrankungen. In einem ersten Projekt werden bei aktuell an Krebs erkrankten Patienten und solchen, deren Diagnose und Behandlung schon länger zurückliegen, pandemiebedingte Veränderungen der Versorgung sowie Auswirkungen auf die gesundheitsbezogene Lebensqualität und die finanzielle Situation verglichen. $\mathrm{Zu}$ diesen Frage- stellungen soll zudem über einen längeren Zeitraum eine Erhebung in vier Wellen im Abstand einiger Monate den Vergleich zwischen Krebspatienten und einer gematchten Kontrollgruppe nicht an Krebs Erkrankter führen. Diese Untersuchungen werden, so die Erwartung, repräsentative Ergebnisse zu den über die medizinische Versorgung hinausgehenden Auswirkungen der Pandemie für Krebsbetroffene liefern, insbesondere zur psychischen, sozialen und finanziellen Situation und $\mathrm{zu}$ entsprechenden Belastungen. Mehr Wissen auch zu diesen Aspekten kann dazu beitragen, dass Krebspatienten, und alle anderen Kranken, auch in Zeiten wie der „Coronakrise“ eine bedarfsgerechte, qualitativ hochwertige Versorgung und Unterstützung erfahren.

\section{Korrespondenzadresse}

\section{Andrea Gaisser}

Im Neuenheimer Feld 280,

69120 Heidelberg, Deutschland

a.gaisser@dkfz.de

\section{Literatur}

1. Fröhling Stefan, Arndt V et al (2020) Versorgung von Krebspatienten: Corona-Effekt in der Onkologie. Dtsch Ärztebl 117(46):A-2234/B-1893 